\title{
A Modified Preparation Method of Ideal Platelet-Rich Fibrin Matrix From Whole Blood
}

\author{
Mirta Hediyati Reksodiputro ${ }^{1 *}$, Alida Roswita Harahap ${ }^{2}$, Lyana Setiawan ${ }^{3}$ and \\ Mikhael Yosia ${ }^{4}$
}

${ }^{1}$ Division of Facial Plastic \& Reconstructive Surgery, Department of Otorhinolaryngology-Head and Neck Surgery, Faculty of Medicine, Universitas Indonesia, Dr. Cipto Mangunkusumo Hospital, Jakarta, Indonesia, ${ }^{2}$ Department of Clinical Pathology, Faculty of Medicine, Universitas Indonesia, Dr. Cipto Mangunkusumo Hospital, Jakarta, Indonesia, ${ }^{3}$ Department of Clinical Pathology, Rumah Sakit Dharmais Pusat Kanker Nasional, Jakarta, Indonesia, ${ }^{4}$ Faculty of Medicine, Universitas Indonesia, Jakarta, Indonesia

OPEN ACCESS

Edited by:

Robert Campbell,

The University of Utah, United States

Reviewed by:

Navin Mishra,

Indira Gandhi Institute of Medical

Sciences, India

Jose Fabio Lana,

Instituto do Osso e da Cartilagem,

Brazi

${ }^{*}$ Correspondence:

Mirta Hediyati Reksodiputro citamirta@yahoo.com

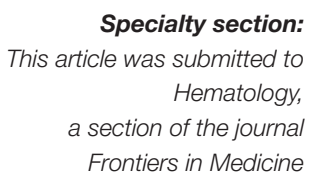

Received: 13 June 2021

Accepted: 07 July 2021

Published: 13 August 2021

Citation:

Reksodiputro MH, Harahap AR,

Setiawan L and Yosia M (2021) A

Modified Preparation Method of Ideal

Platelet-Rich Fibrin Matrix From Whole

Blood. Front. Med. 8:724488.

doi: 10.3389/fmed.2021.724488
One bioproduct that is widely used in the wound healing process is platelet-rich plasma (PRP). PRP is a liquid solution with high autologous platelet concentration, making it a good source of growth factors to accelerate wound healing. Recent development in PRP had created a new product called platelet-rich fibrin matrix (PRFM), which has a denser and more flexible structure. PRFM is the newest generation of platelet concentrate with a fibrin matrix that holds platelet in it. The key concept in creating PRFM from PRP is the addition of $\mathrm{CaCl}_{2}$ followed by centrifugation, which converts fibrinogen to fibrin, and the fibrin cross-links to form a matrix that contains viable platelets. There are many commercially available kits to create PRFM, but they are often expensive and uneconomical. This research will test a modified method of making ideal PRFM from PRP without any commercial kits. The modified method will include determining the minimum level of $\mathrm{CaCl}_{2}$ used, the type of centrifuge, and the speed and duration of centrifugation. By performing a modified preparation method on five samples of whole blood, it was found that the ideal PRFM could be made by mixing PRP with $25 \mathrm{mM} \mathrm{CaCl}_{2}$ and centrifuging it at a speed of 2,264 $\times g$ for 25 min at room temperature. The PRP and PRFM platelet counts of this method tend to be lower than the platelet counts found in other studies. Although visually comparable, further study is needed to compare the performance of PRFMs made with this method and PRFMs made with commercial kits.

Keywords: platelet concentrate, fibrin matrix, platelet rich plasma, platelet rich fibrin matrix, $\mathrm{CaCl}_{2}$, centrifugation

\section{INTRODUCTION}

Otorhinolaryngology specialists in plastic reconstruction have reported successful use of exogenous growth factors and PRP in clinical settings. Sclafani reported that the release of growth factors in wound healing was primarily carried out by platelets, echoing that PRP plays a vital role in wound healing. The influence of growth factors on endothelial cells and fibroblasts will increase within 7 days after injury and disappear after 14 days (1). Other studies have also reported that PRP can increase and accelerate wound healing by $80 \%$ in ulcers compared to placebo $(2,3)$. In clinical settings, people often use PRP in the form of a solution or gel to facilitate tissue repair. The solution or gel form of conventional PRP sometimes poses clinical challenges because it cannot be fixated adequately in certain conditions, such as deep diabetic ulcers with exposed bones and minimal soft tissue availability for PRP injections. 
Platelet-rich fibrin matrix (PRFM) is the latest generation of platelet concentrates with simple preparation without biochemical ingredients (bovine thrombin). PRFM is a slow polymerization of fibrin in PRP, resulting in a PRFM structure that resembles natural fibrin (4). This specific structure will play an essential role in increasing cell migration, cell proliferation, and cyclic formation. Through this polymerization process, all platelets in PRP will be deposited between the PRFM fibrin fibers. According to that logic, levels of platelet (and subsequently, growth factor) in PRFM are expected to be equivalent to PRP.

Laboratory experiments looking at usage of PRFM in specific media show that there was an increase in levels of plateletderived growth factor (PDGF), vascular endothelial growth factor (VEGF), basic fibroblast growth factor (bFGF), and transforming growth factor beta (TGF $\beta$ ) on the first day, followed by a gradual decrease on the next day $(1,5)$. This characteristic was not observed in PRP since most of the growth factors in PRP were released on the first day of application to the wound. PRFM also has fibrin characteristics with a more natural platelet distribution that mimics the body's response to injury and a denser and more flexible macroscopic structure $(6,7)$. These factors theoretically make PRFM superior to use for wound healing.

Commercial kits for preparing PRFM had been available and widely used in clinical settings. One such example is the FIBRINET tubes which can produce PRFM from whole blood through the addition of $\mathrm{CaCl}_{2}$ and centrifugation at $1,100 \times g$ for $6 \mathrm{~min}$ (5). However, the use of the aforementioned commercial tools has several drawbacks, including (1) high prices, rendering the use of PRFM in a clinical setting to be economically dubious; and (2) unknown concentration of platelet in PRFM, increasing the possibility of not achieving the creation of ideal PRFM. This study aims to overcome the problems mentioned above and cover the weaknesses of the existing invention by proposing a modified method to produce PRFM.

In order to guarantee the production of ideal PRFM, the modified method will ensure that platelet-poor plasma (PPP), a by-product in PRFM production, has a platelet content of $0 / \mu \mathrm{l}$, representing the fact that all platelets are attached to the PRFM fibrin matrix at the bottom of the tube. Measurement of TGF $\beta 1$ will be conducted to reassure that growth factors are still available and viable inside the end product. The modified method will also try to make PRFM production more economical by creating PRFM from PRP through improvised methods without using a commercial PRFM production kit. At the same time, this study will determine the minimum amount of $\mathrm{CaCl}_{2}$ needed to produce an ideal PRFM.

This study will carry out trials processing human whole blood, making PRP and adding in $1 \mathrm{M}$ of calcium chloride $\left(\mathrm{CaCl}_{2}\right)$ before centrifuging the solution to form the PRFM. The trials will evaluate whether the modified method can prepare ideal PRFM to accelerate the wound healing process. Improvement in wound healing is essential in reconstructive surgery and will affect the successes of operations both aesthetically and functionally.
TABLE 1 | Profile of volunteers whose blood was used in the study.

\begin{tabular}{llcl}
\hline Subject & Gender & Age (years) & Hematological profile and CBC \\
\hline 1 & Male & 30 & Between normal limits \\
2 & Male & 36 & Between normal limits \\
3 & Male & 41 & Between normal limits \\
4 & Female & 29 & Between normal limits \\
5 & Male & 39 & Between normal limits \\
\hline
\end{tabular}

CBC, Complete Blood Count.

Hematologic profile examinations (platelet count, PT/APTT, bleeding time, clotting time).

\section{MATERIALS AND METHODS}

This study is conducted in Indonesia and approved by the Ethical Clearance Committee from the Faculty of Medicine, University of Indonesia (202/H2.F1/ETIK/2013). The study design and protocol are made and implemented following the Helsinki Declaration. Volunteers recruited from the study were given an explanation, given an information sheet, and signed the informed consent form before participating in the study.

\section{Whole Blood Collection From Participants}

Whole blood samples ( $8 \mathrm{ml}$ per tube) were collected from five healthy volunteers (Table 1). The volunteers are non-smokers, with no history of chronic diseases, and with normal hematologic profile (including normal thrombocyte count, PT/APTT). Blood is collected using the $10 \mathrm{ml}$ vacuum tube with cell selector gel from the PRP kit (RegenKit ${ }^{\circledR}$ A-PRP ${ }^{\circledR}$; Regen Lab, Le Montsur-Lausanne, Switzerland). A small amount of whole blood was also taken to analyze thrombocyte count with the Celtac-a automatic cell counter (Automated Hematology Analyzer MEK6450; Nihon Kohden, Tokyo, Japan). The kit from RegenLab is designed to produce 4-6 mL of PRP from every tube of blood collected. The RegenKit ${ }^{\circledR}$ vacuum tube was inverted back and forth three times after blood collection is finished.

\section{Preparation of PRP and Calculation of Thrombocyte Count From PRP-PPP (Platelet-Poor Plasma)}

This study utilizes a commercial PRP kit, the RegenKit ${ }^{\circledR}$ APRP $^{\circledR}$ (Regen Lab, Le Mont-sur-Lausanne, Switzerland). This instruction directly follows the protocol presented in the kit. PRP can also be produced using other PRP kits or methods. Blood that had been taken from volunteers were immediately centrifuged using RegenLab 642VFD PRP Fixed Angle Centrifuge (Regen Lab, Le Mont-sur-Lausanne, Switzerland). The centrifuge from RegenLab was designed to prepare PRP and was programmed to run at $1,500 \times g$ for $5 \mathrm{~min}$ at room temperature. The centrifuge process resulted in three separate layers: a clear yellowish plasma thrombocyte on top (consisting of a PPP layer on top and a PRP layer in the bottom), a cell selector gel layer with leucocyte in the middle, and a red blood cell layer on the bottom. A small amount of PPP and PRP was taken from each specimen to analyze thrombocyte count using Celtac-a automatic cell counter. $\mathrm{PRP}$ process is determined to be successful when the thrombocyte 
A

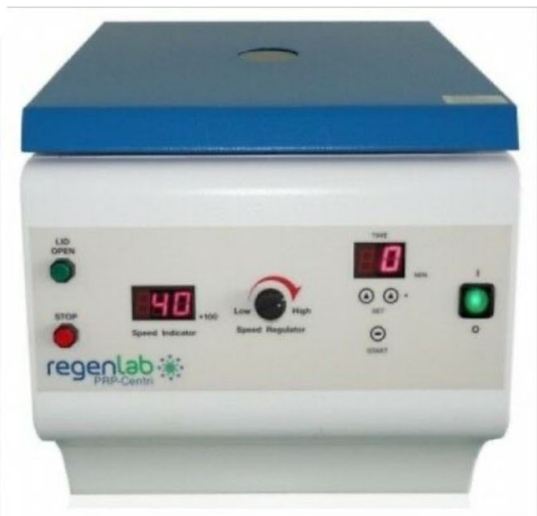

B

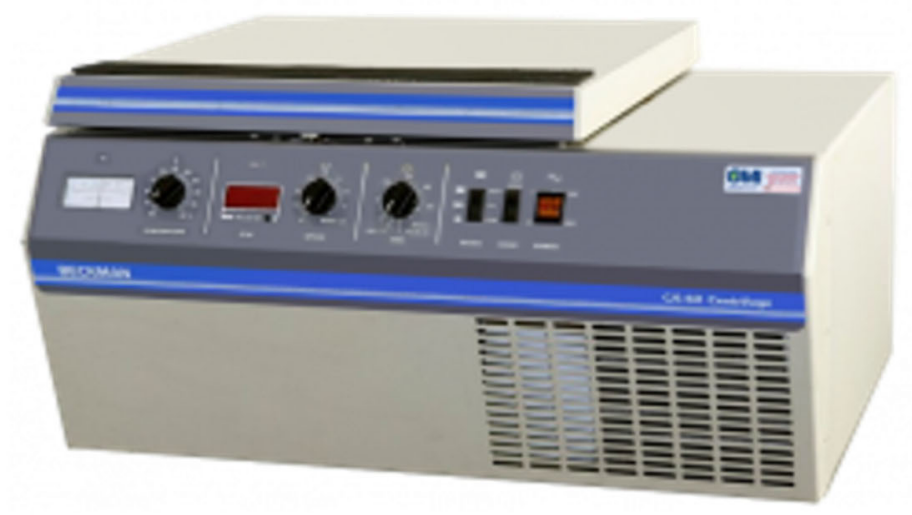

FIGURE 1 | (A) RegenLab PRP-Centri CH-1052 centrifuge used in processing the PRP in cylinder tubes and (B) Beckman CS-6R centrifuge used in processing PRP in a Wheaton bottle.

count in PPP is $0 / \mu$ l. The tube was then inverted gently back and forth three times to mix the plasma, thrombocyte, and leucocyte, forming the final PRP. PPP was mixed with PRP to ensure a larger volume of end product used for PRFM preparation. A total of five PRP specimens will be produced at the end of this step and will be used to produce PRFM.

\section{Preparation of PRFM and Calculation of Thrombocyte Count From PRFM-PPP}

This experiment concentrates on creating PRFM using methods proposed by O'Connel, which is a modified Fibrinet (Cascade Medical Enterprise, Wayne, NJ, USA) PRFM kit method (8). Orthopedic surgeons with more extensive wounds or operating areas usually utilize Fibrinet. Centrifuge from RegenLab was used, as it is the ideal centrifuge to produce PRFM with minimal addition of $\mathrm{CaCl}_{2}$. The maximal speed of this centrifuge is $4,500 \mathrm{RPM}(2,264 \times g)$. Six milliliters of PRP were taken from four different PRP specimens using a micropipette (extra care was given to avoid taking the red blood cell layer) and moved to four separate $10 \mathrm{ml}$ cylindrical centrifuge tubes (Pyrex ${ }^{\circledR}$, Staffordshire, England). Each tube was then added with $\mathrm{CaCl}_{2}$ $1 \mathrm{M}$ until a final concentration of $70,45,25$, or $15 \mathrm{mM}$ of $\mathrm{CaCl}_{2}$ was achieved in each tube. All four tubes were then centrifuged at $2,264 \times g$ for $25 \mathrm{~min}$ at room temperature. The resulting product will consist of two layers, the PRFM and PPP.

PPP from all four PRFM specimens with different concentrations of $\mathrm{CaCl}_{2}$ was then analyzed using the Celtac-a automatic cell counter. As previously mentioned, an ideal PRFM is the one with a $0 / \mu l$ thrombocyte count in their PPP. The lowest concentration of $\mathrm{CaCl}_{2}$ that produces an ideal PRFM was then determined as the minimum recommended concentration of $\mathrm{CaCl}_{2}$.

After the minimum amount of $\mathrm{CaCl}_{2}$ needed had been obtained from the previous steps, the fifth PRP specimen was assigned to an alternative PRFM preparation method to produce a coin-shaped PRFM. This particular shape was sought after because in several reconstructive surgery, a round coin-shaped PRFM with larger surface area is practical in certain clinical conditions. Six milliliters from the fifth PRP specimen was taken using a micropipette and put into a wide-mouth Wheaton bottle (diameter $30 \mathrm{~mm}$ ), followed by addition of the $\mathrm{CaCl}_{2}$. The Wheaton bottle was then centrifuged with a Beckman CS-6R centrifuge at 3,800 RPM $(4,043 \times g)$ for $25 \mathrm{~min}$ (Figure 1). The resulting product consisted of two layers, the PRFM and PPP. The PRFM and PPP were taken for thrombocyte count analysis using the Celtac-a automatic cell counter.

\section{Analysis of the PRFM Characteristic With a Scanning Electronic Microscope (SEM)}

The PRFMs are trimmed into equal sizes, washed using phosphate-buffered saline (PBS), and fixated in $2.5 \%$ glutaraldehyde in PBS for $1 \mathrm{~h}$ at $4^{\circ} \mathrm{C}$. The samples were then washed for the second time with $0.1 \mathrm{M}$ cacodylate buffer ( $\mathrm{pH} 7.3$ ) followed by fixation with $1 \%$ osmium tetroxide (OsO4) and $0.1 \mathrm{M}$ cacodylate buffer for $1 \mathrm{~h}$ at room temperature $\left(22^{\circ} \mathrm{C}\right.$ $\pm 2^{\circ} \mathrm{C}$ ). The samples were then dehydrated in serial ethanol and dried before being placed on an aluminum sheet with silver adhesive paint coated with a layer of $4 \mathrm{nM}$ of gold inside an Edward S150B argon atmosphere apparatus (Crawley, West Sussex, UK). The samples were then observed at $0^{\circ}$ with SEM Stereoscan 200 (Cambridge, UK) at $20 \mathrm{kV}$. Three different fields of view were examined for each sample, and the diameter of thrombocyte and the size of fibrin fibers were examined.

\section{Analysis of TGF $\beta 1$ in PRFM}

TGF $\beta 1$ in PRFM were analyzed using a TGF $\beta 1$ immunoassay kit (Quantikine R\&D Systems; Thermo Fisher Scientific, Portsmouth, NH, USA). The immunoassay kit works by identifying reactions of growth factors in the sample with a TGF $\beta 1$ monoclonal antibody in the microtiter plate wells. The bond between TGF $\beta 1$ and anti-TGF $\beta 1 F$ was identified via a TGF $\beta 1$ polyclonal antibody that is labeled with an enzyme. 
TABLE 2 | Thrombocyte count (per $\mu$ l) for whole blood, PRP, and PPP after preparation of PRP.

\begin{tabular}{lccc}
\hline & \multicolumn{3}{c}{ Thrombocyte count $(/ \mu \mathrm{l})$} \\
\cline { 2 - 4 } Sample & Whole blood & PRP & PRP-PPP \\
\hline Subject 1 & 313.000 & 52.000 & 0 \\
Subject 2 & 414.000 & 64.000 & 0 \\
Subject 3 & 205.000 & 66.000 & 0 \\
Subject 4 & 280.000 & 121.000 & 0 \\
Subject 5 & 393.000 & 186.000 & 0 \\
\hline
\end{tabular}

Note that the thrombocyte count for PPP is $0 / \mu /$ for all the samples, indicating a successful creation of ideal PRP.

With additional substrates, these reactions will produce a varying intensity of colors identified with spectrophotometry at $450 \mathrm{~nm}$. The intensity of the color is directly correlated to the concentration of protein analyzed. Compared to a standard solution with a known concentration, the concentration of TGF $\beta 1$ in the PRFM sample can be analyzed.

PRFM specimens analyzed were put into the microtiter plate wells coated with TGF $\beta 1$ monoclonal antibody and incubated. TGF $\beta 1$ in the sample will bind to the antibody inside the well. After washing the well to remove excess substances, the TGF 1 polyclonal antibody with a horseradish peroxidase (HRP) label was added. Second incubation was done, during which the polyclonal antibody will bind to the anti-TGF $\beta 1^{*}$ TGF $\beta 1$ complex that formed in the first incubation forming a sandwich of antiTGF $\beta 1^{*}$ TGF $\beta 1^{*}$ anti-TGF $\beta 1$.HRPO. A substrate is added to form a blue color that will change into yellow after a stop solution is added. The intensity of color will correlate with the amount of TGF $\beta 1$ inside the sample.

\section{RESULTS}

\section{Volunteer Baseline Data and Examination of PRP}

Blood was drawn from all volunteers and was processed right away using the methods above. All five volunteers had a normal hematologic profile, no chronic comorbidities, and normal CBC (hemoglobin, hematocrit, thrombocyte, leucocyte, differential counts) examinations. Comparison of the amount of thrombocyte in whole blood, PRP, and PPP in the PRP specimen can be seen in Table 2; note that a decrease of thrombocyte count is expected.

\section{Determination of the Minimum Recommended Concentration of $\mathrm{CaCl}_{2}$ for Ideal PRFM}

Four PRP specimen were assigned to different amounts of $\mathrm{CaCl}_{2}$ to determine the minimal amount in which ideal PRFM (the ones with $0 \mu \mathrm{l}$ of thrombocyte in its PPP). The schematic shown in Figure 2 shows that a concentration of $25 \mathrm{mM}$ of $\mathrm{CaCl}_{2}$ is the minimum amount needed to produce ideal PRFM. The fifth specimen was used to produce a coin-shaped PRFM to represent diversity in the practical application of PRFM in different clinical conditions (Figure 3). Both PRP specimen (disc and dome shaped) centrifuged with $25 \mathrm{mM} \mathrm{CaCl}_{2}$ had PPP of $0 / \mu 1$.

\section{PRFM Analysis With SEM}

Measurement of fibrin fiber and platelet diameter in PRFM specimens were performed using SEM. PRFM from five volunteers was prepared and fixated to produces slices that were thin enough to be analyzed by SEM. It was found that PRFM had platelets scattered among the fibrin fibers (Figure 4). The average diameter size (from three measurements in each PRFM specimens) of the subject's platelets and fibrin fiber matrix can be seen in the table below (Table 3). PRFM from Subject 1 dried out during the preparation and fixation process, rendering it inappropriate for analysis.

\section{TGF $\beta 1$ in PRFM}

The average TGF $\beta 1$ rate from PRFM was $37,497 \mathrm{pg} / \mathrm{mg}$, with the highest rate being 42,147 pg/mg and the lowest being 31,849 $\mathrm{pg} / \mathrm{mg}$ (Table 4). PRFM from Subject 1 dried out during the preparation and fixation process, rendering it inappropriate for analysis.

\section{DISCUSSION}

Currently, several methods and commercial kits are available for the preparation of PRP. Most of these methods will produce an end product in liquid or gel form. Due to these mechanical properties, conventional PRP is often impractical in clinical settings that require secure implantation in a specific site or where released growth factors could be washed out during an operation.

The latest development in PRP use for wound healing involves altering its physical property through plasma and platelet stimulation. Alteration of PRP physical properties can be achieved by adding calcium $\left(\mathrm{CaCl}_{2}\right)$ and centrifugation to produce PRFM without the need for additional exogenous thrombin. The addition of $\mathrm{CaCl}_{2}$ and centrifugation to PRP will convert fibrinogen to fibrin, and the fibrin cross-links to form a matrix that contains viable platelets (Figure 4). The PRFM preparation process creates a gel-like matrix containing high concentrations of non-activated, functional, intact platelets within a fibrin matrix. These platelets had been proven to release a relatively constant concentration of growth factors over 7 days (9).

The resulting PRFM is a thin sheet with a more robust physical structure than the liquid PRP. The PRFM can replicate the effect of a natural wound healing response (i.e., the three-dimensional formation of a cross-linked fibrin matrix). This scaffold-like fibrin matrix is essential as a place for platelet adhesion. This scaffolding helps localize platelets and ultimately increases the concentration of growth factors to the desired point or location for tissue regeneration (10).

In this experiment, we obtained a formula for making PRFM without using a costly reagent kit. The ideal PRFM requires that maximal platelets are trapped in the PRFM; the PPP platelet count of $0 / \mathrm{ml}$ can prove this. This experiment also found the 


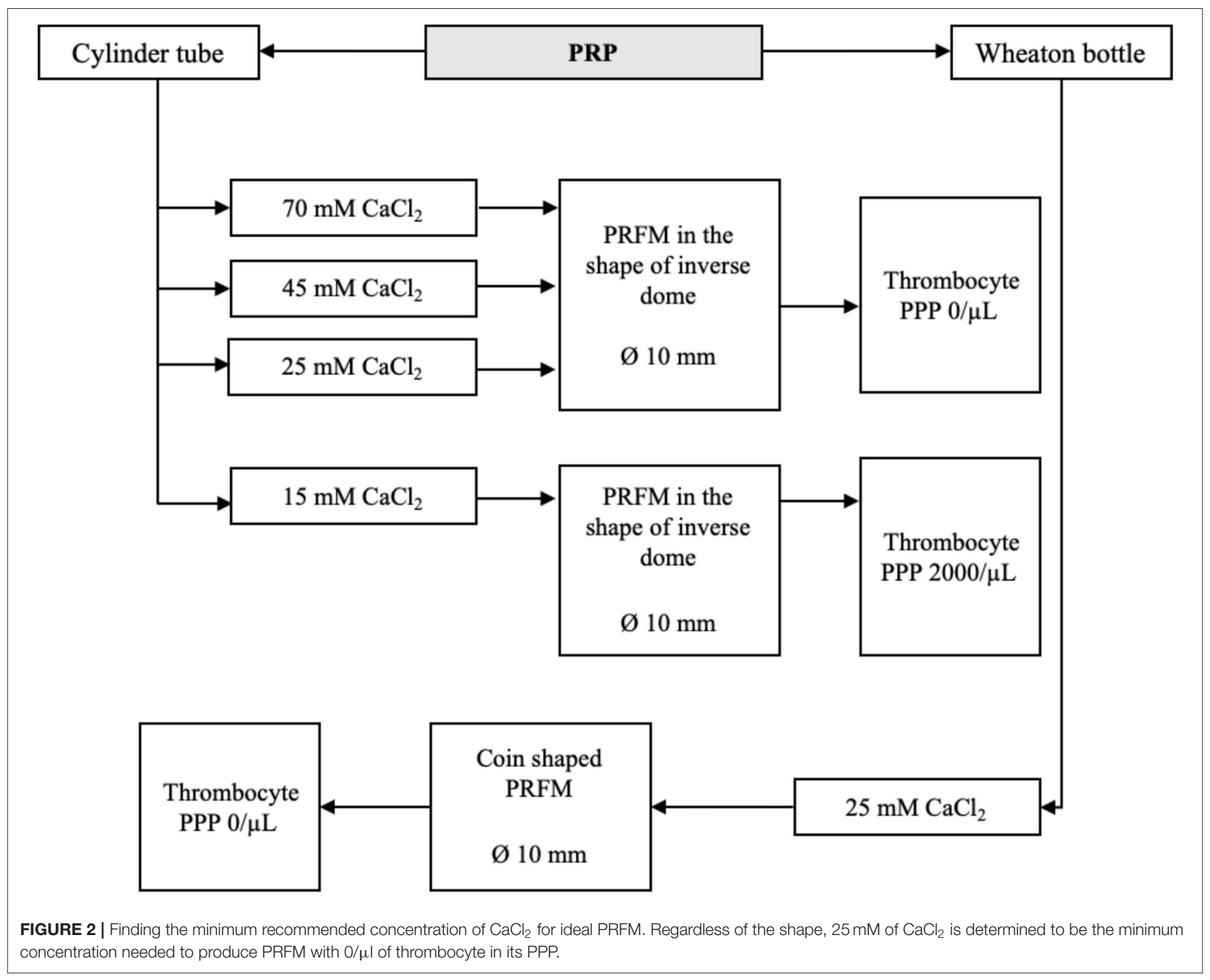

minimum amount of $\mathrm{CaCl}_{2}$ and the centrifuge settings needed to obtain the ideal PRFM. To achieve ideal PRFM, PRP obtained was mixed with $25 \mathrm{mM} \mathrm{CaCl}_{2}$, then centrifuged again at a speed of $2,264 \times g$ for $25 \mathrm{~min}$ at room temperature. The centrifugation will result in two layers, the PPP and PRFM. The platelet level in PPP was $0 / \mu$; thus, it can be assumed that this method produces ideal PRFM with all the platelets from PRP adhering to the matrix fibrin. Commercial kits usually include $\mathrm{CaCl}_{2}$ solutions in their package, though most choose not to disclose the amount or concentration of $\mathrm{CaCl}_{2}$ used in their set.

The PRFM protocol for this study is based on a previous experiment by O'Connell, which breaks down the creation of PRFM from whole blood. O'Connell created PRFM by inserting PRP and $\mathrm{CaCl}_{2}$ in a Wheaton bottle before starting the centrifugation. In his study, $18 \mathrm{ml}$ of whole blood can create $7-8 \mathrm{ml}$ of PRP, which in turn yields a $35 \mathrm{mM}$ round PRFM membrane (8). In our study, $8 \mathrm{ml}$ of whole blood produces $6 \mathrm{ml}$ of PRP, which then yields a $10 \mathrm{~mm}$ round PRFM membrane.
A suspected vital difference in methodology lies during the PRP creation in which tubes containing a thixotropic polyester separator gel was used. These separator gels contain different properties depending on the PRP kit's brand (O'Connell used tubes from the Cascade Autologous System, while this study used one from RegenLab). Differences in the separator gel may affect performance in isolating both the platelets and plasma (containing fibrinogen) from the packed red and white cell fraction $(8,11)$.

The platelet count results for whole blood, PRP and PPP after the first centrifugation showed that the platelet count in PRP was lower than that of the whole blood (Table 2). In general, the preparation of PRP is followed by volume adjustment by removing the $\mathrm{PPP}$, resulting in a platelet concentration $2.5-8$ times higher than that found in whole blood (12). This study's median PRP platelet count was 97,800 (52,000-186,000)/ml-up to $50 \%$ loss of platelet, which is smaller than the typical PRP platelet count (13). The low platelet count is probably caused 


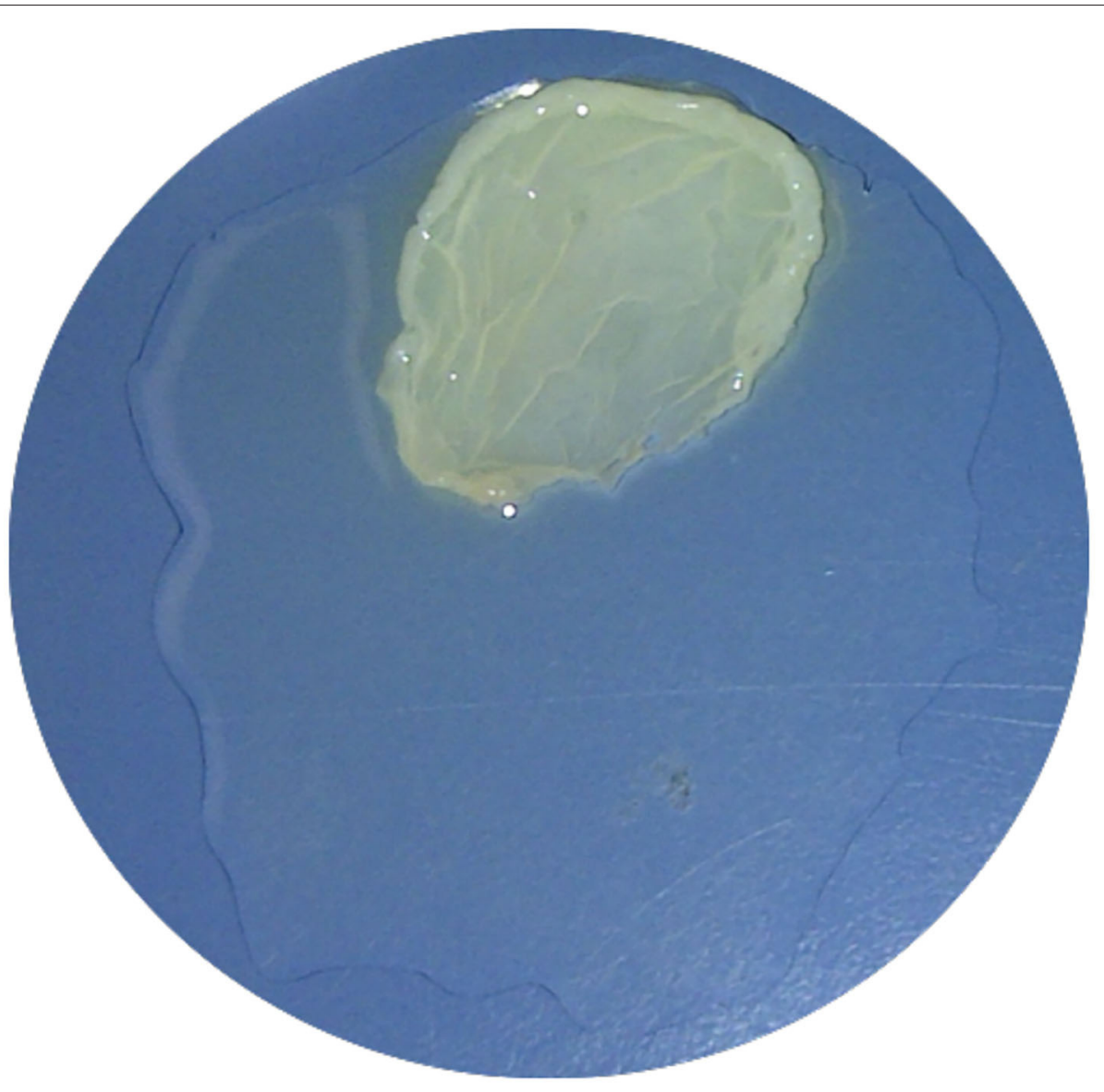

FIGURE 3 | Coin-shaped PRFM produced via centrifuging PRP in a Wheaton bottle.

TABLE 3 | Average fibrin size and platelet diameter in PRFM analyzed through SEM.

\begin{tabular}{lcc}
\hline PRFM & Fibrin fiber size $\mathbf{( n m )}$ & Platelet diameter $(\mathbf{n m})$ \\
\hline Subject 1 & $*$ & $*$ \\
Subject 2 & 446.7 & 670.1 \\
Subject 3 & 403.6 & 638.2 \\
Subject 4 & 360.2 & 625.4 \\
Subject 5 & 428.1 & 638.2 \\
\hline
\end{tabular}

${ }^{\star}$ PRFM dried out during specimen preparation.

by the trapping of platelets in the RegenKit gel tube or due to erythrocyte deposits (Figure 5). Platelet yield must be taken into account in assessing the PRP creation methods used, including standardized methods (commercial kits). Ideally, efforts are made so that minimal platelets are lost since these platelets are a source of growth factors.

Studies that looked upon platelet, leucocyte, and erythrocyte yield on PRP production shed more light on the conundrum regarding the number of platelets being yielded. A review by
TABLE 4 | Level of TGF $\beta 1$.

\section{PRFM}

$\operatorname{TGF} \beta 1(p g / m g)$

\section{Subject 1}

Subject 2

39,796

Subject 3

Subject 4

34,128

Subject 5

${ }^{\star}$ PRFM dried out during specimen preparation.

Marxref concluded that a platelet count of 1 million in a 6$\mathrm{ml}$ aliquot could be considered a benchmark for therapeutic PRP, again showing that the amount of platelet inside the PRP in our experiment falls short in terms of platelet counts to be considered as benchmark PRP (14). It seems that particular emphasis needs to be put on centrifuge force used to yield optimal PRP. Recommendation of a far less intense centrifugal force: $900 \times g$ for $5 \mathrm{~min}$ for separating centrifuge and 1,500 $\times g$ for $15 \mathrm{~min}$ is best to produce optimal PRP (15). The low platelet yield had been one of the primary concerns in this 


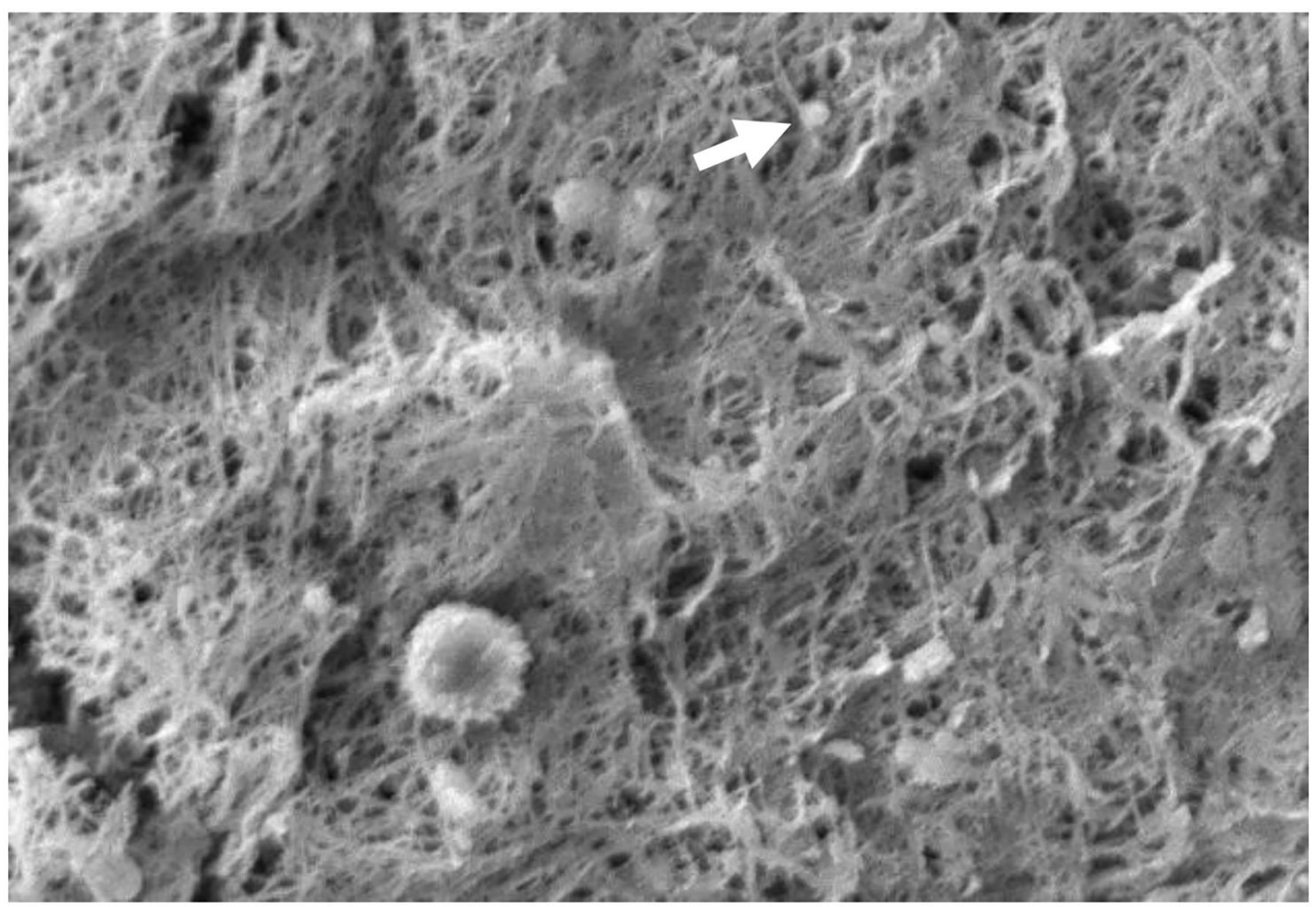

FIGURE 4 | The appearance of fibrin and platelet fibers on PRFM by SEM examination. The white arrows show the platelets between the fibrin fiber matrix. It appears that the fibrin fibers are evenly distributed like a mesh. 1,000 $\times$ magnification.

study; through omitting the dilution of the PRP solution and optimizing centrifugal force, this problem should be resolved. It should be noted, however, that the procedure of creating PRP in this study followed protocols available with the PRP kit; recreation of PRP with other kits or methods may also alleviate this problem.

Based on SEM examination, it can be seen that the PRFM obtained has a microscopic fibrin fiber matrix resembling a mesh (Figure 4). All platelets derived from PRP are evenly distributed among the fibrin fiber matrix. Thus, it can be concluded that this PRFM preparation method consistently produces microscopic fibrin fiber matrix with identical platelet distribution present in ideal PRFM (10). The resulting PRFM is denser and more flexible, resembling a fascial layer that can be sewn, identical to the PRFM preparation resulting from a commercial kit (5).

Other properties analyzed in the PRFM produced are TGF $\beta 1$, a growth factor responsible for controlling and promoting cell growth, proliferation, and differentiation (16). Contrary to popular belief, usually, the TGF $\beta 1$ level in PRP is higher than the PRFM. The difference in TGF $\beta 1$ level can occur due to the activation of platelets by exogenous factors during PRP and PRFM preparation. These exogenous factors can occur in the process of venous blood collection, during pipetting, or in the centrifugation process (17-19). Activated platelets will release granules along with their contents, including growth factors such as TGF $\beta 1$ and PDGF, which will then be dissolved in the plasma. During PRFM preparation, the centrifugation process at $1,800 \times g$ for $60 \mathrm{~min}$ will precipitate platelets and the formed fibrin polymer. The dissolved protein will remain in the plasma, resulting in TGF $\beta 1$ and the activated platelets not precipitating in PRFM. It is known that both cytokines and growth factors secreted from cells have a short half-life, which means that a higher level of TGF $\beta 1$ in PRP does not mean that PRP is better than PRFM (20). Comparison of TGFB1 between PRP and PRFM might not yield any meaningful, practical result, yet it would be interesting to look upon the correlation of the initial amount of TGF $\beta 1$ in PRFM and the amount of TGF $\beta 1$ released after the PRFM had been administered to a media or wound site.

The weakness of this study is a lack of actual observation and comparison toward actual clinical use of the PRFM produced through the modified method proposed. A follow-up study looking at how the PRFM produced through this method fares compared to PRFM produced by commercial kit would further raise the credibility of using the proposed method as an alternative way of preparing PRFM, especially in limited-resource settings. A continuation of the study with more extensive and more varied samples may also yield valuable knowledge and 


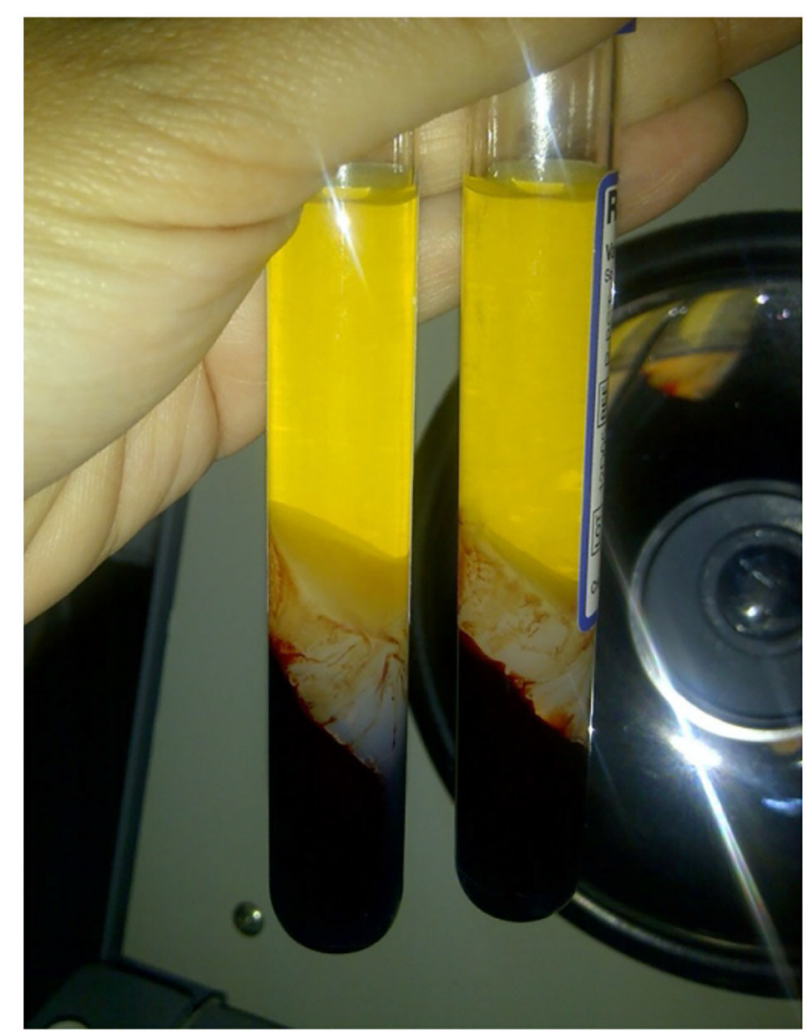

FIGURE 5 | RegenKit tube with separator gel in the middle. Platelet might be trapped in the gel tube thus resulting in lower platelet count in the resulting PRP.

further understanding of the procedure. Further modification and adjustment in the PRP creation method also need to be done to improve platelet yield during PRP production; as

\section{REFERENCES}

1. Sclafani AP. Safety, efficacy, and utility of platelet-rich fibrin matrix in facial plastic surgery. Arch Facial Plast Surg. (2011) 13:247-51. doi: 10.1001/archfacial.2011.3

2. Greer N, Foman NA, MacDonald R, Dorrian J, Fitzgerald P, Rutks I, et al. Advanced wound care therapies for nonhealing diabetic, venous, and arterial ulcers: a systematic review. Ann Intern Med. (2013) 159:532-42. doi: 10.7326/0003-4819-159-8-201310150-00 006

3. Thomas CS, Rao SN, Soni R. The use of autologus platelet-rich plasma in treatment of chronic ulcers and wound. MedPulse Int J Surg. (2020) 13:769. doi: $10.26611 / 10613212$

4. Choukroun J, Diss A, Simonpieri A, Girard MO, Schoeffler C, Dohan SL, et al. Platelet-rich fibrin (PRF): a second-generation platelet concentrate. Part V: histologic evaluations of PRF effects on bone allograft maturation in sinus lift. Oral Surg Oral Med Oral Pathol Oral Radiol Endodontol. (2006) 101:299-303. doi: 10.1016/j.tripleo.2005.07.012

5. Lucarelli E, Beretta R, Dozza B, Tazzari PL, O’Connell SM, Ricci F, et al. A recently developed bifacial platelet-rich fibrin matrix. Eur Cells Mater. (2010) 20:13-23. doi: 10.22203/eCM.v020a02

6. Kobayashi E, Flückiger L, Fujioka-Kobayashi M, Sawada K, Sculean A, Schaller B, et al. Comparative release of growth factors previously stated, omitting dilution, changing the centrifugation speed, or using alternative PRP creation kits or methods might counter this problem. Nevertheless, the study had proven that this modified method could produce ideal PRFM with somewhat comparable quality to the ones produced using a commercial kit.

\section{CONCLUSION}

The proposed modified method by mixing PRP with $25 \mathrm{mM}$ $\mathrm{CaCl}_{2}$ and centrifuging at a speed of 2,264 $\times g$ for $25 \mathrm{~min}$ at room temperature can reliably produce ideal PRFM comparable in quality to the commercial kit. Further follow-up study is needed to compare the performance of PRFM produced by the modified method to those produced with commercial kits.

\section{DATA AVAILABILITY STATEMENT}

The raw data supporting the conclusions of this article will be made available by the authors, without undue reservation.

\section{AUTHOR CONTRIBUTIONS}

MR: study concept, design, ethical submission, data collection, data analysis, interpretation, and writing the paper. AH: study concept, design, lab examination, data analysis, and interpretation. LS: study concept, design, lab examination, and data analysis. MY: data analysis and interpretation and writing the paper and submission. All authors contributed to the article and approved the submitted version.

\section{ACKNOWLEDGMENTS}

The authors would like to thank friends and fellow staff in the University of Indonesia for supporting the study. 20:2353-60. doi: 10.1007/s00784-016-1719-1

7. Gole PV, Muhammed N, Patadia SR. Efficacy of autologous platelet rich fibrin matrix in the management of non-healing ulcers. Int J Res Dermatol. (2019) 5:686. doi: 10.18203/issn.2455-4529.IntJResDermatol20194566

8. O'Connell SM, Impeduglia T, Hessler K, Wang XJ, Carroll RJ, Dardik $\mathrm{H}$. Autologous platelet-rich fibrin matrix as cell therapy in the healing of chronic lower-extremity ulcers. Wound Repair Regen. (2008) 16:74956. doi: 10.1111/j.1524-475X.2008.00426.x

9. Carroll RJ, Arnoczky SP, Ms SG, Ascp MT, Connell SMO. Characterization of autologous growth factors in Cascade $(\mathbb{2}$ Platelet-Rich Fibrin Matrix (PRFM). Processing. (2005) 4-5. https://www.pulsevet.com/Research/PRP/ ResearchArticles/Carroll_Growth\%20Factor_Release_WhitePaper.pdf

10. Roy S, Driggs J, Elgharably $H$, Biswas S, Findley $M$, Khanna S, et al. Platelet-rich fibrin matrix improves wound angiogenesis via inducing endothelial cell proliferation. Wound Repair Regen. (2011) 19:753-66. doi: 10.1111/j.1524-475X.2011.00740.x

11. O'Connell SM, Hessler K, Dardik H. Cascade (R) autologous system plateletrich fibrin matrix in the treatment of chronic leg ulcers. Adv Wound Care. (2012) 1:52-5. doi: 10.1089/wound.2011.0290

12. Cole BJ, Seroyer ST, Filardo G, Bajaj S, Fortier LA. Platelet-rich plasma: where are we now and where are we going? Sports Health. (2010) 2:20310. doi: $10.1177 / 1941738110366385$ 
13. Straum OK. The optimal platelet concentration in platelet-rich plasma for proliferation of human cells in vitro-diversity, biases, and possible basic experimental principles for further research in the field: a review. PeerJ. (2020) 8:e10303. doi: $10.7717 /$ peerj.10303

14. Marx RE. Platelet-rich plasma: evidence to support its use. J Oral Maxillofac Surg. (2004) 62:489-96. doi: 10.1016/j.joms.2003.12.003

15. Delong JM, Russell RP, Mazzocca AD. Platelet-rich plasma: the PAW classification system. Arthrosc J Arthrosc Relat Surg. (2012) 28:9981009. doi: 10.1016/j.arthro.2012.04.148

16. Massagué J, Chen YG. Controlling TGF- $\beta$ signaling. Genes Dev. (2000) 14:627-44. doi: 10.1101/gad.14.6.627

17. Eppley BL, Woodell JE, Higgins J. Platelet quantification and growth factor analysis from platelet-rich plasma: implications for wound healing. Plast Reconstr Surg. (2004) 114:1502-8. doi: 10.1097/01.PRS.0000138251.07 040.51

18. Bagamery K, Kvell K, Barnet M, Landau R, Graham J. Are platelets activated after a rapid, one-step density gradient centrifugation? Evidence from flow cytometric analysis. Clin Lab Haematol. (2005) 27:75-7. doi: 10.1111/j.1365-2257.2004.00662.x

19. Laffan MA, Manning RA. Investigation of haemostasis. In: Dacie and Lewis Practical Haematology, 12th ed. London: Churchill Livingstone (2017). p. 366409.
20. Ren X, Zhao M, Lash B, Martino MM, Julier Z. Growth factor engineering strategies for regenerative medicine applications. Front Bioeng Biotechnol. (2020) 7:469. doi: 10.3389/fbioe.2019.00469

Conflict of Interest: The authors declare that the research was conducted in the absence of any commercial or financial relationships that could be construed as a potential conflict of interest.

Publisher's Note: All claims expressed in this article are solely those of the authors and do not necessarily represent those of their affiliated organizations, or those of the publisher, the editors and the reviewers. Any product that may be evaluated in this article, or claim that may be made by its manufacturer, is not guaranteed or endorsed by the publisher.

Copyright (c) 2021 Reksodiputro, Harahap, Setiawan and Yosia. This is an openaccess article distributed under the terms of the Creative Commons Attribution License (CC BY). The use, distribution or reproduction in other forums is permitted, provided the original author(s) and the copyright owner(s) are credited and that the original publication in this journal is cited, in accordance with accepted academic practice. No use, distribution or reproduction is permitted which does not comply with these terms. 